\title{
Strictly invariant submodules
}

\author{
Simion Breaz, Grigore Călugăreanu and Andrey Chekhlov *
}

February 5, 2019

\begin{abstract}
If $M$ is an $R$-module, we study the submodules $K \leq M$ with the property that $K$ is invariant with respect to all monomorphisms $K \rightarrow M$. Such submodules are called strictly invariant. For the case of $\mathbb{Z}$-modules (i.e. Abelian groups) we prove that in many situations these submodules are invariant with respect to all homomorphisms $K \rightarrow M$, submodules which were called strongly invariant.
\end{abstract}

\section{Introduction}

Let $K$ be a submodule of a module $M$, and let $\mathcal{X}$ be a class of homomorphisms such that $f(K)$ makes sense for all $f \in \mathcal{X}$. We say that $K$ is invariant with respect to the class $\mathcal{X}$ if the inclusion $f(K) \leq K$ holds for all $f \in \mathcal{X}$. For instance, $K$ is fully invariant, injective invariant, respectively characteristic, if $K$ is invariant with respect to that class $\mathcal{X}$, where $\mathcal{X}$ is $\operatorname{End}(M), \operatorname{Mon}(M)$ (i.e. the set of all monic endomorphisms of $M$ ), respectively $\operatorname{Aut}(M)$. In module theory there are important classes of modules which can be characterized by the invariance of some submodules with respect to some classes of homomorphisms. For instance, a module $M$ is quasi-injective (pseudo-injective) if and only if it is fully invariant (characteristic) as a submodule of the injective hull of $M$, cf. [13] (respectively [12]). We refer to [19] for some general statements about modules which are invariant with respect to classes of endomorphisms of injective hulls.

Injective invariant subgroups of Abelian groups were termed S-characteristic and left invariant, respectively, in [2] or [16]. These were used in [4] for the study of (co)hopfian modules.

The submodules $K$ which are invariant with respect to $\mathcal{X}=\operatorname{Hom}(K, M)$ are called strongly invariant, and these are studied in [6], with a special attention to the case of Abelian groups. We will say that the submodule $K$ of $M$ is strictly invariant if it is invariant with respect to the set $\mathcal{X}=\operatorname{Mon}(K, M)$ of all monomorphisms $K \rightarrow M$. Clearly, strongly invariant submodules are strictly invariant and strictly invariant submodules are characteristic.

*2010 AMS Subject Classification: 16D10, 16D80,20K27 Key words: strictly invariant submodule, strongly invariant submodule, Abelian group, strictly invariant subgroup 
For reader's convenience we mention that the same notions are discussed in the case of non-Abelian groups in 22, where strongly invariant (normal) subgroups are termed homomorph containing and strictly invariant subgroups are termed isomorph containing.

In the next section we study general properties of strictly invariant submodules. Among these it is proved that the set of all strictly invariant submodules of a module is a complete lattice with respect to the inclusion relation, Proposition 10. Moreover, if the additive group of the module has no elements of order 2, then every strictly invariant submodule is invariant with respect to idempotent endomorphisms, Proposition 17.

In the third section we study strictly invariant subgroups of Abelian groups. We mention that in Example 4 it is proved that there exist strictly invariant submodules which are not strongly invariant. However, we were not able to construct such an example for the case of Abelian groups. Therefore we are focussed on finding conditions (as general as possible) on the group and/or on the subgroup, which imply that the strictly invariant subgroups are strongly invariant, in order to argue the enunciation of the following conjecture: every strictly invariant subgroup of an Abelian group is strongly invariant. Very large classes of Abelian groups are shown to support this conjecture.

In this context we mention that in the case of Abelian groups, all pseudoinjective groups are quasi-injective, 21]. A similar situation occurred in [4]: denoting by $\mathcal{Q}(G)$, the family of all subgroups $N \leq G$ such that every homomorphism $N \longrightarrow G$ extends to an endomorphism of $G$ and by $\mathcal{P}(G)$, the family of all subgroups $N \leq G$ such that every injective homomorphism $N \longrightarrow G$ extends to an endomorphism of $G$, though we strongly suspect that $\mathcal{Q}(G)=\mathcal{P}(G)$ for Abelian groups, the proof which shows that finitely generated subgroups from $\mathcal{P}(G)$ are also in $\mathcal{Q}(G)$ was already very hard (and the general question is still open).

Notice that for noncommutative groups it is easy to give examples of strictly invariant subgroups which are not strongly invariant: the dihedral 2-groups of order at least 8 and the infinite dihedral group. The order 8 group $D_{8}$ has $a$ unique cyclic maximal subgroup $H$ (of order 4) which clearly is strictly but not strongly invariant in $D_{8}$. Indeed, there are other two order 4 subgroups which are Klein, and all the other order 2 subgroups are (clearly) cyclic.

We finally mention that, starting from [3, Dikranian, Giordano Bruno, Goldsmith, Salce, Virili and Zanardo defined and studied fully inert subgroups of Abelian groups in [8 - 11, [17, [18. Replacing fully invariant subgroups by strongly invariant subgroups, led the first and second authors to study the strongly inert subgroups of Abelian groups in [5]. A natural continuation of all these (kindly suggested by the referee) would be to study the strictly inert subgroups and compare these with strongly inert subgroups. We postpone this to a forthcoming paper.

All modules we consider are over a unital ring denoted $R$. $\mathbb{F}_{2}$ denotes the field with two elements and $\mathbb{Z}(2)$ the Abelian group with two elements. For other notations for Abelian groups we refer to [14] and [15]. 


\section{General properties}

Using the above definitions we obtain the following chart

$$
\begin{array}{ccccc}
\begin{array}{c}
\text { strongly-invariant } \\
\Downarrow(4)
\end{array} & \stackrel{(*)}{\Longrightarrow} & \text { fully-invariant } & & \\
\text { strictly-invariant } & \stackrel{(1)}{\Longrightarrow} & \text { injective-invariant } & \stackrel{(3)}{\Longrightarrow} & \text { characteristic }
\end{array}
$$

The following examples (the numbering corresponds to these) show that all reversed implications fail $((2)$ presents fully invariant subgroup which is not strictly invariant; as for $(*)$, such examples are given in [6]).

First, an injective invariant subgroup which is not strictly invariant.

Example 1 Let $G=\left\langle a_{1}\right\rangle \oplus\left\langle a_{2}\right\rangle \oplus\left\langle a_{3}\right\rangle$ with $o\left(a_{i}\right)=2^{i}$, and $H=\left\langle 2 a_{2}\right\rangle \oplus\left\langle a_{1}+\right.$ $\left.2 a_{3}\right\rangle$. Since $G$ is finite, characteristic and injective invariant subgroups coincide (because injective functions from $G$ to $G$ are bijective). It is proved in [15, p. 9] that $H$ is characteristic, and it is easy to see that $H$ is not strictly invariant (e.g. take $2 a_{2} \longmapsto a_{1}$ and $a_{1}+2 a_{3} \longmapsto a_{2}$ ). Moreover, it is not fully invariant.

Example 2 If $p$ is a prime, the subgroup $p \mathbb{Z}$ of $\mathbb{Z}$ is not strictly invariant but it is fully invariant.

Other examples may be found in [6] or [7].

Next, a characteristic subgroup which is not injective invariant.

Example 3 By [1, Theorem 2.14], for every prime $p$ there exists a torsion free Abelian group $G$ of rank 2 with endomorphism ring isomorphic to $R=\mathbb{Z}[\sqrt{-p}]$. Since the units of $R$ are \pm 1 , it follows that all subgroups are characteristic. Moreover, $\mathbb{Q} \otimes R$ is a division ring, hence all non-zero endomorphisms of $G$ are injective. Let $x \in G$ be a non-zero element, and let $f$ and $g$ be two endomorphisms of $G$ which are $\mathbb{Q}$-independent in $\mathbb{Q} \otimes R$. Suppose $f(x)$ and $g(x)$ are not $\mathbb{Z}$-independent. Then there exist two non-zero integers $m$ and $n$ such that $m f(x)=n g(x)$ and so $m f-n g$ is not injective. Hence $m f=n g$, a contradiction. Thus for every non-zero element $x$ of $G$, the subgroup $R x$ has to be of rank 2 , hence the subgroup $\langle x\rangle$ is not injective invariant.

Next, we present an example of strictly invariant submodule which is not strongly invariant.

Example 4 Let $R$ be a ring such that there exist non-isomorphic simple modules $S_{1}, S_{2}$ and $T$ such that

1. the endomorphism rings of these modules are isomorphic to $\mathbb{Z}_{2}$;

2. there are non-splitting exact sequences

$$
0 \rightarrow S_{1} \rightarrow K \rightarrow T \rightarrow 0, \text { and } 0 \rightarrow S_{2} \rightarrow K \rightarrow T \rightarrow 0 .
$$


By using the pullback diagram

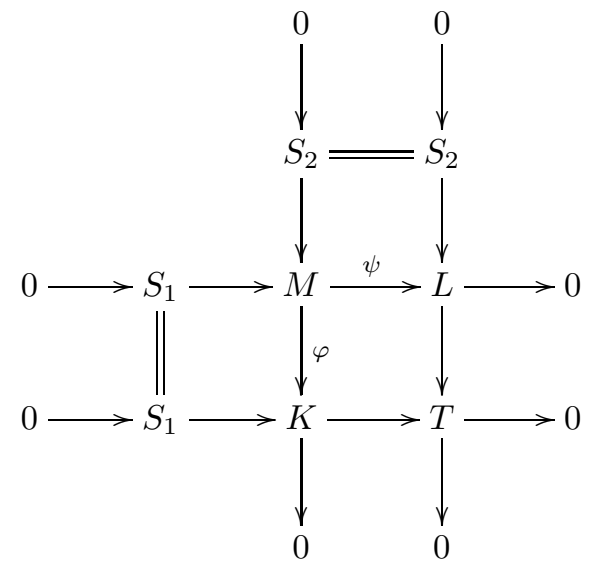

we construct a module $M$ such that the set of its submodules is $\left\{0, S_{1}, S_{2}, S_{1} \oplus\right.$ $\left.S_{2}, M\right\}$ and $M / S_{1} \oplus S_{2} \cong T$ (this module is also used in [20, Lemma 2]).

Let $\varphi: M \rightarrow K$ be a non-zero homomorphism. Then $\varphi\left(S_{2}\right)=0$. If $\varphi\left(S_{1}\right)=$ 0 then $\varphi$ induces a non-zero homomorphism $T \rightarrow M$, which is impossible. We obtain that $\varphi\left(S_{1}\right) \neq 0$, and it follows that $\left.\varphi\right|_{S_{1}}$ is the inclusion map. Therefore, if $\varphi_{1}, \varphi_{2}: M \rightarrow K$ are two non-zero homomorphisms then the restriction of these homomorphisms to $S_{1} \oplus S_{2}$ coincide. It follows that $\left(\varphi_{1}-\varphi_{2}\right)\left(S_{1} \oplus S_{2}\right)=0$, hence $\varphi_{1}=\varphi_{2}$. This way $\operatorname{Hom}(M, K)=\{0, \varphi\}$ and in the same way we obtain $\operatorname{Hom}(M, L)=\{0, \psi\}$.

It is easy to see that if $\rho: M \rightarrow K \times L$ is the homomorphism induced by $\varphi$ and $\psi$ then $\rho$ is a monomorphism. Since $\operatorname{Hom}(M, K \times L) \cong \operatorname{Hom}(M, K) \times$ $\operatorname{Hom}(M, L)$, it follows that $\rho$ is the only monomorphism from $M$ into $K \times L$. We conclude that $\rho(M)$ is strictly invariant. Since there exists an epimorphism $\mathrm{M} \rightarrow \mathrm{K}$, and $K \times 0$ is not contained in $\rho(M)$, it follows that $\rho(M)$ is not strongly invariant.

For reader's convenience we recall the concrete example described in [19, Example 3.1].

Let $R=\left(\begin{array}{ccc}\mathbb{F}_{2} & \mathbb{F}_{2} & \mathbb{F}_{2} \\ 0 & \mathbb{F}_{2} & 0 \\ 0 & 0 & \mathbb{F}_{2}\end{array}\right)$. Then the right $R$-module $M=\left(\begin{array}{ccc}\mathbb{F}_{2} & \mathbb{F}_{2} & \mathbb{F}_{2} \\ 0 & 0 & 0 \\ 0 & 0 & 0\end{array}\right)$

satisfies the required conditions: the simple submodules are $S_{1}=\left(\begin{array}{ccc}0 & \mathbb{F}_{2} & 0 \\ 0 & 0 & 0 \\ 0 & 0 & 0\end{array}\right)$ and $S_{2}=\left(\begin{array}{ccc}0 & 0 & \mathbb{F}_{2} \\ 0 & 0 & 0 \\ 0 & 0 & 0\end{array}\right)$, and it is easy to see that the simple R-module $M /\left(S_{1} \oplus S_{2}\right)$ is not isomorphic to $S_{1}$ or $S_{2}$. 
In what follows we study the basic properties of strictly invariant submodules. First observe that strict invariance is not a transitive property.

Example 5 In order to show this, we observe that if $S$ and $T$ are non-isomorphic simple modules, $0 \rightarrow S \rightarrow K \rightarrow T \rightarrow 0$ is a non-splitting exact sequence and $M=K \oplus S$, then $S \oplus 0$ is strictly invariant in $K \oplus 0, K \oplus 0$ is strictly invariant in $M$, but $S \oplus 0$ is not strictly invariant in $M$.

For the case of Abelian groups, consider $G=H \oplus L=\mathbb{Z}\left(2^{\infty}\right) \oplus \mathbb{Z}(2)$ with $K=\mathbb{Z}(2)<H$. Then $K=S(H)$, the socle, is strongly and so strictly invariant in $H$. It is not strictly invariant in $G$, since the composition of the isomorphism $K \cong L$ with the injection $\iota_{L}: L \longrightarrow G$ does not map $K$ into $K$. Finally, $H$ is a fully invariant direct summand - as divisible part of $G$ - and so strongly and strictly invariant in $G$.

Next, if $H \leq L \leq M$ and $H$ is strictly invariant in $M$ then $L$ might not be strictly invariant in $M$.

Example 6 It suffices to take $K$ as in Example 5, $M=K \oplus K, H=S \oplus S$ and $K=K \oplus S$.

Proposition 7 Let $M$ be a module and let $H \leq K$ be submodules of $M$. If $H$ is strictly invariant in $M$ and $K / H$ is strongly invariant in $M / H$, then $K$ is strictly invariant in $M$.

Proof. Let $f: K \longrightarrow M$ be an injective homomorphism. Since $H$ is strictly invariant in $M$, the map $\widetilde{f}: K / H \longrightarrow M / H, \widetilde{f}(k+H)=f(k)+H$ is well-defined and a homomorphism. Since $K / H$ is strongly invariant in $G / H, \widetilde{f}(K / H) \subseteq$ $K / H$ which shows that $f(K) \subseteq K$.

Notice that we cannot weaken the hypothesis " $K / H$ is strongly invariant in $M / H$ " only to strictly invariant, as the example below shows .

If $H \leq K \leq M$ and $K$ is strictly invariant in $M$ then $K / H$ might not be strictly invariant in $M / H$.

Example 8 For instance, if $M=H \oplus K=\mathbb{Z}_{2} \oplus \mathbb{Z}_{4}$, the socle $H+2 K$ is strictly invariant in $M$ but $(H+2 K) / 2 K=\mathbb{Z}_{2}$ is not strictly invariant in $M / 2 K=\mathbb{Z}_{2} \oplus \mathbb{Z}_{2}$.

Further, the intersection of a family of strictly invariant submodules is not (in general) strictly invariant.

Example 9 In order to prove this, we use the same module as in Example 5 . It is easy to see that the socle $S \oplus S$ of $M$ and $K$ are strictly invariant submodules of $M$, but $S \oplus 0=(S \oplus S) \cap K$ is not strictly invariant.

For the case of Abelian groups we can consider $G=D \oplus R$, where $D$ is a divisible $p$-group and $R$ is a reduced $p$-group. Then $D$ and $G[p]=D[p] \oplus R[p]$ are strongly invariant subgroups; however, the subgroup $D \cap G[p]=D[p]$ is not 
strictly invariant in $G$ (this covers the missing example in [6], where an example of two strongly invariant subgroups with not strongly invariant intersection was not given).

Intersections of strictly invariant subgroups may not be strictly invariant also in torsion-free groups. To see this we use Example 2 (p. 107, [7]). We recall some details about this example.

Let $E_{1}, E_{2}, E_{3}$ and $E_{4}$ be torsion-free groups of rank 1, let $p, q, p_{2}$ and $p_{3}$ be distinct primes, let the types of the groups $E_{1}, E_{2}$, and $E_{3}$ be pairwise incomparable, and let $E_{1} \cong E_{4}, p_{2} E_{2}=E_{2}, p_{3} E_{3}=E_{3}, p E_{1} \neq E_{1}, p E_{2} \neq E_{2}$, $p E_{3} \neq E_{3}, p_{2} E_{1} \neq E_{1}, p_{2} E_{3} \neq E_{3}, p_{3} E_{1} \neq E_{1}, p_{3} E_{2} \neq E_{2}, q E_{1} \neq E_{1}$, $q E_{2} \neq E_{2}, q E_{3} \neq E_{3}$.

A group $G$ is constructed as subgroup of a divisible torsion-free group, using a vector space over the field of rational numbers. Write $A=\left\langle E_{1}, E_{2}, p^{-\infty}\left(e_{1}+e_{2}\right)\right\rangle$, $B=\left\langle E_{3}, E_{4}, q^{-\infty}\left(e_{3}+e_{4}\right)\right\rangle$ and $G=A \oplus B$ where $0 \neq e_{i} \in E_{i}, i \in\{1,2,3,4\}$ and $p^{-\infty} a$ is the infinite set $p^{-1} a, p^{-2} a, \ldots$ If $\mathbf{t}\left(E_{i}\right)$ denotes the type of $E_{i}$, it is shown that $A$ and $E_{1} \oplus E_{4}=G\left(\mathbf{t}\left(E_{1}\right)\right)$ are strongly invariant in $G$ but $E_{1}=A \cap G\left(\mathbf{t}\left(E_{1}\right)\right) \cong E_{4}$ is not strictly invariant.

In the sequel we prove some basic properties of strictly invariant submodules. We denote by $\mathcal{T}(M)$ the set of all strictly invariant submodules of $M$.

Proposition 10 Let $M$ be an $R$-module. If $\left\{S_{i}\right\}_{i \in I}$ is a family of submodules from $\mathcal{T}(M)$ then $\sum_{i \in I} S_{i} \in \mathcal{T}(M)$. Consequently, $(\mathcal{T}(M), \subseteq)$ is complete lattice.

Proof. Let $\left\{S_{i}\right\}_{i \in I}$ be a family of strictly invariant submodules of a module $M$ and let $f: \sum_{i \in I} S_{i} \longrightarrow M$ be an injective homomorphism. Denoting by $\iota_{i}: S_{i} \longrightarrow \sum_{i \in I} S_{i}(i \in I)$ the inclusions, the compositions $f \circ \iota_{i}: S_{i} \longrightarrow G$ are also injective. By hypothesis, $\left(f \circ \iota_{i}\right)\left(S_{i}\right) \subseteq S_{i}$ and so $f\left(\sum_{i \in I} S_{i}\right) \subseteq \sum_{i \in I} S_{i}$, as required.

The existence of inf's now follows because an ordered set $A$ is a complete lattice if and only if for every subset $B \subseteq A$, there exists $\sup B$.

Using Example 9] we observe that in general the infimum of a family $\left\{S_{i}\right\}_{i \in I}$ from $\mathcal{T}(M)$ is not the intersection of these submodules, that is, the complete lattice $(\mathcal{T}(M), \subseteq)$ above is not a complete sublattice of the lattice of all submodules of $M$.

Let $M$ be an $R$-module. If $K \leq M$, we denote by $\mathcal{M}_{M}(K)$ the sum of all submodules $f(K)$, where $f$ ranges all monomorphisms $f: K \rightarrow M$. We denote by $\mathcal{S}(M)$ the lattice of all submodules of $M$ and $\mathcal{M}_{M}(\mathcal{S}(M))=\left\{\mathcal{M}_{M}(K)\right.$ : $K \leq M\}$.

Proposition 11 Let $M$ be an $R$-module. Then $\mathcal{M}_{M}(-): \mathcal{S}(M) \rightarrow \mathcal{S}(M)$ is an idempotent decreasing operator, and $\mathcal{M}_{M}(\mathcal{S}(M))=\mathcal{T}(M)$. 
Proof. If $f: \mathcal{M}_{M}(K) \rightarrow M$ is an injective homomorphism, then for every monomorphism $g: K \rightarrow M$, since $g(K) \leq \mathcal{M}_{M}(K) \leq M$, we can consider $f \circ g: K \rightarrow M$, which is also a monomorphism. Then $(f \circ g)(K) \leq \mathcal{M}_{M}(K)$, and we conclude that $f\left(\mathcal{M}_{M}(K)\right) \leq \mathcal{M}_{M}(K)$. Finally for every submodule $K$ of $M$ we have $\mathcal{M}_{M}(K) \in \mathcal{T}(M)$ and the surjectivity follows from the fact that $K \in \mathcal{T}(M)$ clearly implies $\mathcal{M}_{M}(K)=K$.

Corollary 12 Let $M$ be an $R$-module and $K \leq M$.

1. If $f: K \rightarrow M$ is a homomorphism and $f(K) \nsubseteq \nsubseteq \mathcal{M}_{M}(K)$ then for every $\alpha: K \rightarrow \mathcal{M}_{M}(K)$ there exists $x \in K$ such that $f(x)=\alpha(x)$.

2. $f(K) \cap \mathcal{M}_{M}(K) \neq 0$ for every $0 \neq f \in \operatorname{Hom}(K, G)$.

3. If $H \leq M$ is a submodule of $M$ such that $H \cap \mathcal{M}_{M}(K)=0$, then $\operatorname{Hom}(H, K)=0$.

Proof. 1. The image of $f-\alpha$ is not contained in $\mathcal{M}_{M}(K)$. Then $f-\alpha$ is not a monomorphism.

For 2 and 3 we apply 1 taking for $\alpha$ the inclusion map.

Corollary 13 Let $H$ be a strictly invariant submodule of $M$. Then:

1. $f(H) \subseteq H$ for every non-zero homomorphism $f: H \rightarrow G$ such that $f(f(H) \cap H)=0$.

2. $f(H) \cap H \neq 0$ for every $0 \neq f \in \operatorname{Hom}(H, M)$.

3. $\operatorname{Hom}(H, L)=0$ for every $L \leq M$ such that $L \cap H=0$.

Proof. 1. Suppose there exists $f: H \rightarrow G, f \neq 0$, such that $f(f(H) \cap H)=0$ and consider $\bar{f}: H \rightarrow G, \bar{f}(h)=h+f(h)$ for every $h \in H$. If $h+f(h)=0$ then $h \in f(H) \cap H$, hence $f(h)=0$ and so $h=0$. Therefore, $\bar{f}$ is a monomorphism and $f(H) \subseteq H$ by strictly invariance.

The statements 2 and 3 are consequences of 1 .

Corollary $14 \mathrm{~A}$ direct summand is strictly invariant if and only if it is fully invariant.

For any pair $A, N$ of modules, denote by $S_{A}(N)=\sum_{f \in \operatorname{Hom}(N, A)} f(N)$ the $N$-socle of $A$, a submodule of $A$.

Proposition 15 Let $M=A \oplus B$ be an $R$-module. If $K \leq A$ and $L \leq B$ are submodules such that $K \oplus L$ is strictly invariant in $M$ then

1. $K$ is strictly invariant in $A$.

2. $L$ is strictly invariant in $B$.

3. $S_{A}(L) \leq K$ and $S_{B}(K) \leq L$. 
Proof. Let $f: K \rightarrow A$ be a monomorphism. Then $\bar{f}=f \oplus \iota_{L}: K \oplus L \rightarrow A \oplus B$ is a monomorphism ( $\iota_{N}: L \rightarrow B$ denotes the inclusion map) and, since $K \oplus L$ is strictly invariant, the inclusion $f(K) \leq K$ follows.

Any homomorphism $f: L \rightarrow A$ induces a homomorphism $\bar{f}: K \oplus L \rightarrow A \oplus B$, $\bar{f}(k+\ell)=k+f(\ell)+\ell$ for every $k \in K, \ell \in L$. If $k+f(\ell)+\ell=0$ then $\ell=0$ and we also obtain $k=0$. Therefore, $\bar{f}$ is injective, and it is easy to see that $f(\ell) \in K$ for every $\ell \in L$.

In the following result, for any (finite or infinite) cardinal $k, M^{(k)}$ denotes the direct sum of $k$ copies of $M$.

Corollary 16 Let $H$ be a strictly invariant submodule of $M$. Then the following conditions are equivalent:

1) $H^{2}$ is a strictly invariant submodule of $M^{2}$.

2) $H^{(k)}$ is a strictly invariant submodule of $M^{(k)}$ for every cardinal number $k$.

3) $H^{(k)}$ is a strongly invariant submodule of $M^{(k)}$ for every cardinal number $k$.

4) $H$ is a strongly invariant submodule of $M$.

Proof. 1) $\Rightarrow$ 4) Follows from Proposition 15

$4) \Rightarrow 3$ ) Since $H$ is strongly invariant in $M$, by [6], $H^{(k)}$ is strongly invariant in $M^{(k)}$ for any finite $k$. For any infinite cardinal $k$, since every element of $M^{(k)}$ belongs to a direct summand isomorphic to $M^{(n)}$ for some finite $n, H^{(k)}$ is also strongly invariant in $M^{(k)}$.

3) $\Rightarrow 2$ ) Obvious.

2) $\Rightarrow$ 1) Obvious.

Proposition 17 Let $M=A \oplus B$ be a module such that the additive group $A$ has no elements of order 2 . If a submodule $H \leq M$ is strictly invariant then there exist $K \leq A, L \leq B$ such that

1) $H=K \oplus L$.

2) $K$ is strictly invariant in $A$ and $L$ is strictly invariant in $B$.

3) $S_{A}(L) \leq K$ and $S_{B}(K) \leq L$.

Proof. By Proposition 15, it suffices to prove that $H=K \oplus L$ with $K \leq A$ and $L \leq B$.

Let $\pi_{A}: M \rightarrow A$ and $\pi_{B}: M \rightarrow B$ be the projections and suppose $H$ is strictly invariant in $M$. If $\pi \in\left\{\pi_{A}, \pi_{B}\right\}$ and $\pi(H) \not \subset H$, there is $0 \neq h \in H$ such that $\pi(h) \notin H$. Therefore $(\pi+1)(h) \notin H$ and so the restriction $\left.(\pi+1)\right|_{H}$ is not injective (otherwise $H$ is not strictly invariant). Hence $\operatorname{ker}\left(\left.(\pi+1)\right|_{H}\right) \neq 0$ and if $\pi(x)=-x$ for $0 \neq x \in H$ then $\pi(x)=-\pi(x)$, i.e. $2 \pi(x)=0$, a contradiction.

It follows that $\pi_{A}(H) \leq H$, and similarly $\pi_{B}(H) \leq H$. Therefore $H=$ $M \oplus N$, where $M=\pi_{A}(H) \leq A$ and $N=\pi_{B}(H) \leq B$.

Remark 18 The previous proposition is not valid if both $A$ and $B$ have elements of order 2 .

This follows from the construction used in Example 4 


\section{Strictly invariant subgroups}

As mentioned in the Introduction, for fairly large classes of groups, we show that our conjecture, "strictly invariant subgroups of Abelian groups are strongly invariant", holds.

We start the investigation of strictly invariant subgroups of Abelian groups with a consequence of Proposition 15 (in this section, unless otherwise stated, "group" means "Abelian group"). By $\mathbb{P}$ we denote the set of all prime numbers and for an Abelian group $G$ and a prime $p, G_{p}=\left\{x \in G: \exists n \in \mathbb{N}, p^{n} x=0\right\}$ denotes the $p$-component of $G$. For an element $x \in G$, the $p$-height of $x$, denoted $h_{p}(x)$, is the smallest integer $n$ such that $x \in p^{n} H$. If $x \in p^{n} H$ for all positive integers $n$ then we say that $x$ is of infinite height.

If $G$ is an Abelian group, we denote by $D(G)$ its divisible part. Moreover, if $p$ is a prime then $D_{p}(G)$ denotes the $p$-component of $D(G)$.

Corollary 19 Let $H$ be a strictly invariant subgroup of a group $G$. Then

1. $D(H)=D(G)$ whenever $D(H)$ is not a torsion group.

2. $D_{p}(H)=D_{p}(G)$ whenever $p$ is a prime and $D_{p}(H) \neq 0$.

Proof. We chose a decomposition $H=H_{0} \oplus D(H)$. Using [14, Theorem 21.2], we can find a direct decomposition $G=K \oplus D(H)$ such that $H_{0} \leq K$. By Proposition 15] $S_{K}(D(H)) \leq H_{0}$. Since $H_{0}$ is reduced and every image of a divisible group is divisible, it follows that $S_{K}(D(H))=0$.

1. If $D(H)$ is not torsion then it has a direct summand isomorphic to $\mathbb{Q}$, hence for every non-reduced group $L$ we have non-zero homomorphisms $D(H) \rightarrow$ $L$. It follows that $K$ is reduced, hence $D(H)=D(G)$.

2. If $D_{p}(H) \neq 0$ then $D(H)$ has a direct summand isomorphic to $\mathbb{Z}\left(p^{\infty}\right)$. From $S_{K}(D(H))=0$ it follows that $D_{p}(K)=0$, hence $D_{p}(H)=D_{p}(G)$.

Theorem 20 Let $G$ be a group and let $H$ be a p-subgroup of $G$. Then $H$ is strictly invariant in $G$ if and only if it satisfies one of the following conditions:

1. $H=G_{p}$.

2. there exists a non-negative integer $n$ such that $H=G\left[p^{n}\right]$.

3. there exists a non-negative integer $n$ such that $H=G\left[p^{n}\right]+D_{p}(G)$.

Proof. Suppose $H$ is strictly invariant. Since $H$ is a $p$-group, we can suppose that $G$ is also a $p$-group.

As in the proof of Corollary 19 we can find direct decompositions $G=$ $K \oplus D(H)$ and $H=H_{0} \oplus D(H)$ with $H_{0} \leq K$. Using Proposition 15 it follows that $H_{0}$ is strictly invariant in $K$. Therefore, we can assume w.l.o.g. that $H$ is reduced.

Case I: $H$ is not bounded. We will prove that $H=G$. Let us fix an element $y \in G$. We can assume w.l.o.g that there exists $u$, the smallest positive integer such that $p^{u} y \in H$. 
If $p^{u} y=0$, we chose $\langle x\rangle$ a direct summand of $H$ such that $\operatorname{ord}(x) \geq \operatorname{ord}(y)$. If $H=\langle x\rangle \oplus L$ then $\langle x+y\rangle+L=\langle x+y\rangle \oplus L$ and $\operatorname{ord}(x+y)=\operatorname{ord}(x)$. It follows that $\langle x+y\rangle+L \cong H$, and we obtain that $x+y \in H$, hence $y \in H$.

Suppose that $p^{u} y \neq 0$ and we can find non-zero elements in $\left\langle p^{u} y\right\rangle$ whose heights computed in $H$ are infinite. Let $\oplus_{n>0} B_{n}$ be a basic $p$-subgroup of $H$, where for every $n$ the group $B_{n}$ is isomorphic to a direct sum of cyclic groups of order $p^{n}$ (see [14, Theorem 32.4]). Since $H$ has an unbounded basic subgroup, there exists $n>0$ such that $p^{n}>\operatorname{ord}(y)$ and $B_{n} \neq 0$. Since all non-zero elements of $B_{n}$ are of finite height, it follows that $\left\langle p^{u} y\right\rangle \cap B_{n}=0$, hence we can find a $B_{n}$-high subgroup $C \leq H$ such that $\left\langle p^{u} y\right\rangle \leq C$. By the proof of [14, Proposition 27.1] it follows that $H=B \oplus C$. Therefore, there exists a decomposition $H=\langle x\rangle \oplus L$ such that $\operatorname{ord}(x) \geq \operatorname{ord}(y)$ and $p^{u} y \in L$. Write $H=\langle x\rangle \oplus L$, and consider the homomorphism $f: H \rightarrow G, f(m x+\ell)=m x+m y+\ell$, for all $m \in \mathbb{Z}$ and $\ell \in L$. If $k x+k y+\ell=0$, it follows that $k y \in H$, hence $p^{u}$ divides $k$. Therefore $k y+\ell \in L$, hence $k x=0$. Since $\operatorname{ord}(x) \geq \operatorname{ord}(y)$ we obtain $k y=0$, and it follows that $\ell=0$. Finally, $f$ is a monomorphism and it is easy to conclude that $y \in H$.

If $p^{u} y \neq 0$ and all non-zero elements of $\left\langle p^{u} y\right\rangle$ are of finite heights (computed in $H$ ) then by [14, Theorem 33.4], there exists a basic subgroup $B$ of $H$ such that $\left\langle p^{u}\right\rangle \leq B$. Since $B$ is unbounded, we can find a cyclic direct summand $\langle x\rangle$ of $B$, hence of $G$, such that $\operatorname{ord}(x) \geq \operatorname{ord}(y)$, and $\langle x\rangle \cap\left\langle p^{u} y\right\rangle=0$. Then there exists a decomposition $H=\langle x\rangle \oplus L$ such that $p^{u} y \in L$, and we can repeat the proof used in the previous case to conclude that $y \in H$.

Case II: $H$ is bounded. If $p^{n}=\exp H$ then clearly $H \leq G\left[p^{n}\right]$. Assume that $H<G\left[p^{n}\right]$ and let $x \in H$ be such that $\operatorname{ord}(x)=p^{n}$. Since $G\left[p^{n}\right]$ is generated by the elements of order $p^{n}$ in $G$, there exists $y \in G\left[p^{n}\right]$ such that $\operatorname{ord}(y)=p^{n}$ and $y \notin H$. By [14, Theorem 27.1], there exist $K, L \leq G$ such that $G\left[p^{n}\right]=\langle x\rangle \oplus K=\langle y\rangle \oplus L$. By Dedekind's law, $H=H \cap G\left[p^{n}\right]=\langle x\rangle \oplus(H \cap K)$. Since $L \cong K$, there exists $L_{1} \leq L$ such that $L_{1} \cong H \cap K$ and it follows that $\langle y\rangle \oplus L_{1}$ and $\langle x\rangle \oplus(H \cap K)=H$ are isomorphic. Since $y \notin H$ this contradicts the fact that $H$ is strictly invariant in $G$. Thus $H=G\left[p^{n}\right]$, as desired.

As for the converse, it is enough to observe that if $H$ verifies any of the conditions $1-3$ then it is strongly invariant.

Remark 21 For the case $p \neq 2$ the above result can also be proved by using Proposition 17.

Namely, if $H$ has an unbounded basic subgroup, we write $G=D_{p}(G) \oplus R$ with reduced $R$ and $H=D_{p}(G) \oplus K$ with $K \leq R$. Let $k$ be a positive integer. There exists a cyclic direct summand $C \cong \mathbb{Z}\left(p^{n}\right)$ of $R$ with $n \geq k$. Applying Proposition 17 for the direct decomposition $G=\left(D_{p}(G) \oplus C\right) \oplus L$, it follows that $S_{L}\left(D_{p}(G) \oplus C\right)=S_{L}(C) \leq H$. It is easy to see that $G\left[p^{n}\right] \leq H$, hence $G\left[p^{k}\right] \leq H$ for all $k$ and the proof is complete.

From now on, starting with the next corollary, the results are all in the line of the conjecture, stating that every strictly invariant subgroup of an Abelian group is strongly invariant. 
First we are able to show that

Corollary 22 Every torsion strictly invariant subgroup of any group is strongly invariant.

Proof. It is proved in [6] that a torsion subgroup is strongly invariant if and only if all its primary components are strongly invariant. Using Theorem 20. the conclusion now follows. Indeed, all subgroups from the theorem, $G_{p}, G\left[p^{n}\right]$ and $G\left[p^{n}\right]+D_{p}(G)$ are strongly invariant.

In the following proposition, $r_{p}(K)$ denotes the $p$-rank of $K$. We will prove that if the divisible part of a group is large enough, then all strictly invariant subgroups are strongly invariant.

Proposition 23 Let $G=D(G) \oplus R$ be a group and $r_{p}(D(G)) \geq \max \left\{r_{p}(R), \aleph_{0}\right\}$ for every $p \in \mathbb{P} \cup\{0\}$. Then every strictly invariant subgroup of $G$ is strongly invariant.

Proof. Let $H$ be a strictly invariant subgroup of $G$. Then $H=D(G) \oplus K$ (we use Corollary (19) and we can suppose that $K \leq R$. It suffices to prove that $K$ is strongly invariant in $R$. In order to do this, let us fix a homomorphism $f: K \rightarrow R$.

By the rank hypotheses it follows that $K$ can be embedded in $D(G)$ and $D(G) \cong D(G) \oplus D(G)$. Therefore, there exists a monomorphism $\alpha: H \rightarrow D(G)$. We consider the homomorphism $g: H \rightarrow G$, defined by $g(d+k)=\alpha(d+k)+f(k)$ for all $d \in D(G)$ and $k \in K$. It is easy to see that $g$ is a monomorphism. Therefore $f(k) \in K$ for all $k \in K$ and the proof is complete.

The following results refer to torsion-free subgroups or torsion-free groups.

Proposition 24 Let $H$ be a strictly invariant torsion-free subgroup of a group $G$. If $H$ is of finite rank then it is strongly invariant.

Proof. Let $f: H \rightarrow G$ be a homomorphism. We claim that there exists a positive integer $k$ such that for all $x \in H$ we have $f(x) \neq k x$.

By contradiction suppose that the above claim fails. Then for every positive integer $k$ there exists $x_{k} \in H$ such that $f\left(x_{k}\right)=k f\left(x_{k}\right)$. We will prove by induction on the cardinality of $S$ that every non-empty finite subset $S \subseteq\left\{x_{k}\right.$ : $\left.k \in \mathbb{N}^{\star}\right\}$ is linearly independent. Since for $|S|=1$ the property is obvious, suppose that all non-empty subsets $S \subseteq\left\{x_{k}: k \in \mathbb{N}^{\star}\right\}$ of cardinality at most $n$ are linearly independent. Let $\left\{x_{k_{1}}, \ldots, x_{k_{n+1}}\right\}$ be a subset of cardinality $n+1$, and suppose that there exist integers $\alpha_{1}, \ldots, \alpha_{n+1}$ such that $\sum_{i=1}^{n+1} \alpha_{i} x_{k_{i}}=0$. Applying $f$ we obtain $\sum_{i=1}^{n+1} k_{i} \alpha_{i} x_{k_{i}}=0$, and so $\sum_{i=1}^{n}\left(k_{i}-k_{n+1}\right) \alpha_{i} x_{k_{i}}=0$. By the induction hypothesis it follows that $\left(k_{i}-k_{n+1}\right) \alpha_{i}=0$ for all $i \in\{1, \ldots, n\}$, and now it is easy to conclude that $\left\{x_{k_{1}}, \ldots, x_{k_{n+1}}\right\}$ is linearly independent. Hence the rank of $H$ is infinite, a contradiction. 
Let $k$ be a positive integer such that for all $x \in H$ we have $f(x) \neq k x$. Then the map $g: H \rightarrow G, g(x)=k x+f(x)$ is a monomorphism. Using the strictly invariance of $H$, it follows that $g(H) \subseteq H$, hence $f(H) \subseteq H$, and the proof is complete.

Proposition 25 If $G$ is torsion-free and all rank 2 pure subgroups of $G$ are indecomposable, then every strictly invariant subgroup of $G$ is strongly invariant.

Proof. Suppose there exists a non-injective homomorphism $f: H \rightarrow G$. Then there is a non-zero element $x \in H$ such that $f(x)=x$. Indeed, let $f \in \operatorname{Hom}(H, G)$ and $f(H) \not \leq H$. If $g$ is the embedding of $H$ in $G$ then $(f-g) H \not \leq H$, so there exists a non-zero $x \in H$ such that $f(x)=x$. Take $y$ a non-zero element from the kernel of $f$, and let $L$ be the pure subgroup generated by $x$ and $y$. For every non-zero element $z \in L$, we have a relation $k z=m x+n y$ with $k \neq 0$. Then $k f(z)=m x$, and so $k f^{2}(z)=m x=k f(z)$. From the torsion-free hypothesis, we can view $f$ as an idempotent endomorphism of $L$ whose image has rank 1 . It follows that $L$ is not indecomposable, a contradiction.

Examples of such groups include the purely indecomposable groups determined by Griffith in the reduced case (see Theorem 88.5 [15]) and in particular the so-called cohesive groups considered by Dubois (see Exercise 17, § 88, [15]).

Next, it is easy to see that the only strictly invariant subgroups of rank 1 torsion-free groups are the trivial ones (i.e. 0 or the whole group). This is clear for $\mathbb{Z}$ and follows from Corollary 19 for $\mathbb{Q}$. By Theorem 20, this also holds for $\mathbb{Z}(p)^{\mathbb{N}}$.

Proposition 26 A subgroup of a completely decomposable group is strictly invariant if and only if it is a fully invariant direct summand.

Proof. Let $G=\oplus_{i \in I} G_{i}$ be a completely decomposable group, where all groups $G_{i}$ are of rank 1. If $H$ is a strictly invariant subgroup of $G$, then using Proposition 17 it follows that for all $i \in I$ we have $\pi_{i}(H) \leq H$, where $\pi_{i}: G \rightarrow G_{i}$ denotes the projection. Then $H=\oplus_{i \in I}\left(H \cap G_{i}\right)$, and, using Proposition 15 , $H \cap G_{i}$ is a strictly invariant subgroup of $G_{i}$ for every $i \in I$. By the preceding paragraph, $H=\oplus_{j \in J} G_{j}$, where $J$ is the set of all $j \in I$ such that $H \cap G_{j}=G_{j}$. The conclusion is now a consequence of Corollary 14.

Corollary 27 Let $G$ be a separable torsion-free group and $H$ a nonzero strictly invariant subgroup. Then $H$ is strongly invariant.

Proof. Let $f: H \longrightarrow G$ be a homomorphism. If $x \in H$ then there exists a finite rank completely decomposable $G_{1} \oplus \cdots \oplus G_{n}$ direct summand of $G$ such that $x, f(x) \in G_{1} \oplus \cdots \oplus G_{n}$. Using Proposition 17 it follows that $K=$ $H \cap\left(G_{1} \oplus \cdots \oplus G_{n}\right)$ is a strictly invariant subgroup of $G_{1} \oplus \cdots \oplus G_{n}$. Then by Proposition 26] $K$ is strongly invariant, whence $f(x) \in H$. 
Finally, we show that the groups, all whose subgroups are strictly invariant, coincide with those all whose subgroups are strongly invariant.

Theorem 28 The only groups in which every subgroup is strictly invariant are the direct sums of cocyclic groups, at most one, for each prime number.

Proof. The proof in [6] holds verbatim with obvious changes for torsion groups. Indeed, a subgroup $H$ of a torsion group $G$ is strictly invariant if and only if the $p$-component $H_{p}$ is strictly invariant in $G_{p}$ for each prime $p$, in a $p$-group $G$ every subgroup is strictly invariant if and only if $G$ is cocyclic, and, in a torsion group every subgroup is strictly invariant if and only if each $p$-component has this property.

Further, there are no torsion-free nor (genuine) mixed groups with only strictly invariant subgroups. Indeed, using the multiplication with $\frac{1}{p}$ for a suitable prime $p$, it is easy to see that rank 1 torsion-free groups are strictly invariant simple (i.e. have only trivial strictly invariant subgroups). Therefore, no torsion-free groups have only strictly invariant subgroups.

As for (genuine) mixed groups $G$, the torsion part $T(G)$ must be as in the strongly invariant case and so is a direct summand (see [6]). If $G=T(G) \oplus F$, we continue with $F$ as above.

Acknowledgement. Thanks are due to the referee, for corrections and suggestions which improved our presentation.

\section{References}

[1] D. M. Arnold Finite rank torsion free Abelian groups and rings. Lecture Notes in Mathematics 931. Berlin-Heidelberg-New York. Springer-Verlag, 1982.

[2] R. Baer Groups without isomorphic proper subgroups. Bull. Amer. Math. Soc. 50 (4) (1944), 267-278.

[3] V. V. Belyaev, M. Kuzucuoglu, E. Seckin Totally inert groups. Rend. Sem. Mat. Univ. Padova 102 (1999), 151-156.

[4] S. Breaz, G. Călugăreanu, P. Schultz Subgroups which admit extensions of homomorphisms. Forum Mathematicum 27 (5) (2015), 2533-2549.

[5] S. Breaz, G. Călugăreanu Strongly inert subgroups of Abelian groups. Rend. Sem. Mat. Univ. Padova 138 (2018), 101-114.

[6] G. Călugăreanu Strongly invariant subgroups of Abelian groups. Glasgow J. Math. 57 (2) (2015), 431-443.

[7] A. R. Chekhlov On Strongly Invariant Subgroups of Abelian Groups. Mathematical Notes 102 (1) (2017), 105-110. 
[8] U. Dardano, D. Dikranjan, S. Rinauro. Inertial properties in groups. International J. of Group Theory 7 (3) (2018), 17-62.

[9] D. Dikranjan, A. Giordano Bruno, L. Salce, S. Virili. Intrinsic algebraic entropy. J. of Pure and Applied Algebra 219 (7) (2015), 2933-2961.

[10] D. Dikranjan, A. Giordano Bruno, L. Salce, S. Virili. Fully inert subgroups of divisible Abelian groups. J. Group Theory 16 (6) (2013), 915-940.

[11] D. Dikranjan, L. Salce, P. Zanardo. Fully inert subgroups of free Abelian groups. Periodica Math. Hungarica 69 (2014), 69-78.

[12] N. Er, S. Singh, A. K. Srivastava Rings and modules which are stable under automorphisms of their injective hulls. J. Algebra 379 (2013), 223-229.

[13] C. Faith Lectures on Injective Modules and Quotient Rings. Lecture Notes in Mathematics 49, Springer Verlag, 1967.

[14] L. Fuchs Infinite Abelian Groups. vol. 1 Academic Press (1970).

[15] L. Fuchs Infinite Abelian Groups. vol. 2 Academic Press (1973).

[16] B. Goldsmith, K. Gong A note on Hopfian and co-Hopfian Abelian groups. (2012), Proceedings of the Conference on Group Theory and Model Theory, Contemporary Maths. Series, editors: M. Droste, L. Fuchs, L. Strüngmann, K. Tent.

[17] B. Goldsmith, L. Salce, P. Zanardo. Fully inert submodules of torsion-free modules over the ring of p-adic integers. Colloquium Math. 136 (2014), 169-178.

[18] B. Goldsmith, L. Salce, P. Zanardo. Fully inert subgroups of Abelian pgroups. J. of Algebra 419 (2014), 332-349.

[19] P. A. Guil Asensio, T. C. Quynh, A. K. Srivastava Additive unit structure of endomorphism rings and invariance of modules. Bull. Math. Sci. 7 (2017), $229-246$.

[20] S. K. Jain, S. Singh Quasi-injective and pseudo-injective modules. Can. Math. Bull. 18 (1975), 359-366.

[21] S. K. Jain, S. Singh On pseudo-injective modules and self-pseudo-injective rings. J. Math. Sciences 2 (1967), 23-31.

[22] http://groupprops.subwiki.org/wiki/Homomorph-containing_subgroup

S. Breaz, Department of Mathematics, Babeş-Bolyai University, 1 Kogălniceanu Street, Cluj-Napoca, Romania

E-mail: bodo@math.ubbcluj.ro 
G. Călugăreanu, Department of Mathematics, Babeş-Bolyai University, 1 Kogălniceanu Street, Cluj-Napoca, Romania

E-mail: calu@math.ubbcluj.ro

A. Chekhlov, Faculty of Mechanics and Mathematics, Tomsk State University, Tomsk, Russia

E-mail: cheklov@math.tsu.ru 